\title{
A two-photon excitation study on the role of carotenoid dark states in the regulation of plant photosynthesis
}

\author{
Axel Wehling · Peter J. Walla
}

Published online: 13 February 2007

(C) Springer Science+Business Media B.V. 2007

\section{Erratum to: Photosynth Res (2006) 90:101-110 \\ DOI 10.1007/s11120-006-9088-2}

In this article Equation 2 must be

$F^{\mathrm{TPE}}(t)=I_{\mathrm{Abs}}^{\mathrm{TPE}} \cdot \Phi_{\mathrm{Transfer}}(t) \cdot \Phi_{\mathrm{Fl}}(t)$.

instead of

$F^{\mathrm{TPE}}(t)=I_{\mathrm{Abs}}^{\mathrm{TPE}} \cdot \Phi_{\mathrm{Fl}}(t)$

In this article important details of Fig. 1 are incorrect or missing. The correct Fig. 1 is presented below:

\section{Reference}

Holt NE, Zigmantas D, Valkunas L, Li X-P, Niyogi KK, Fleming GR (2005) Carotenoid cation formation and the regulation of photosynthetic light harvesting. Science 307:433-436

The online version of the original article can be found at http://dx.doi.org/10.1007/s11120-006-9088-2

\footnotetext{
A. Wehling · P. J. Walla

Department for Biophysical Chemistry, Technical

University of Brunswick, Institute for Physical and

Theoretical Chemistry, Hans-Sommerstr. 10, D-38106

Braunschweig, Germany

P. J. Walla $(\square)$

Department for Spectroscopy and Photochemical Kinetics,

Max-Planck-Institute for Biophysical Chemistry,

Am Faßberg 11, D-37077 Göttingen, Germany

e-mail: pwalla@gwdg.de
} 


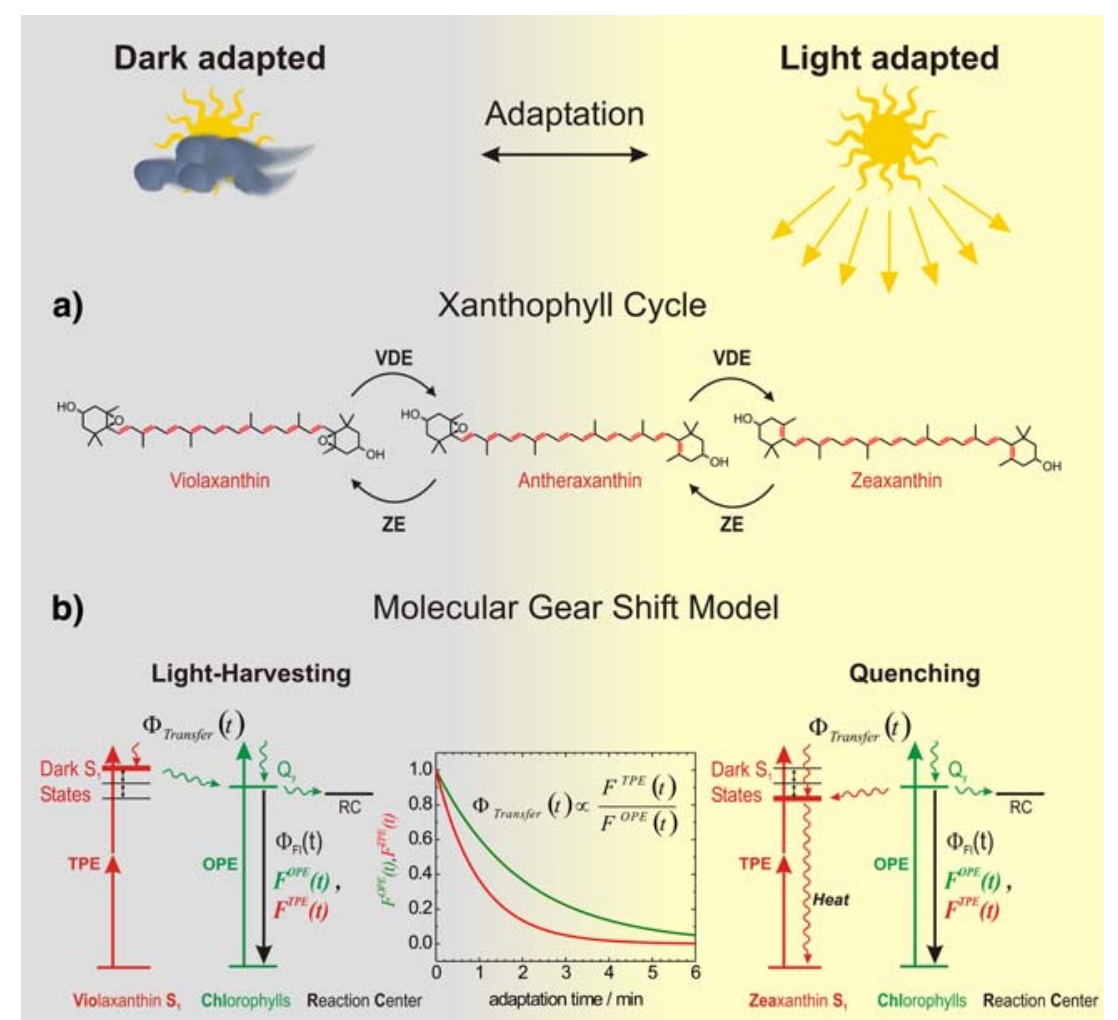

c)

Proposed Summarized Model
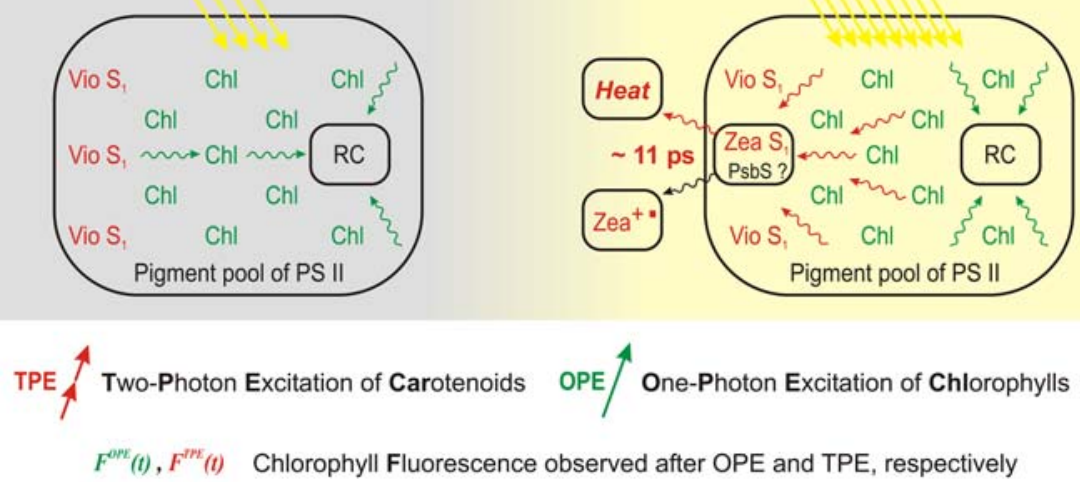

$\leadsto$ Light harvesting $\leadsto$ Quenching and heat dissipation $\leadsto$ Radical cation formation

Fig. 1 (a) In the xanthophyll cycle the enzymes violaxanthin deepoxidase (VDE) and zeaxanthin epoxidase (ZE) vary the amount of conjugated double bonds depending on the light conditions. (b) In the molecular gear shift model the change in the conjugation length causes a drop in the dark state energy of Zea forming an effective excitation energy trap. The quenching reduces the chlorophyll fluorescence, $F^{\mathrm{OPE}}(t)$, observed with conventional one-photon excitation (OPE). The carotenoid dark states can only be accessed by two-photon excitation (TPE). Differences in the OPE- and TPE-sensitized chlorophyll fluorescence kinetics, $F^{\mathrm{OPE}}(t)$ and $F^{\mathrm{TPE}}(t)$, reflect a change in the energy transfer coupling, $\phi_{\text {Transfer }}(t)$. (c) Proposed model for the biophysical quenching mechanism: In the same way a single reaction centre (RC) can act as an effective trap for excitation energy of the entire light-harvesting pigment pool under low light conditions even a single zeaxanthin molecule potentially suffices to act as an effective quenching trap. Once the carotenoid dark state of Zea has been populated it can convert the energy effectively into heat with in about 11 ps or additionally into a long living ( $\sim 150 \mathrm{ps})$ carotenoid radical cation during the same time period (Holt et al., 2005) 